\title{
INVESTIGATION OF MIXING EFFICIENCY AND PRESSURE DROP IN T-SHAPED MICROMIXERS
}

\author{
A.S. Lobasov ${ }^{1,2}$, A.V. Minakov ${ }^{1,2}$, V.V. Kuznetsov ${ }^{2}$, V.Ya. Rudyak ${ }^{3}$, A.A. Shebeleva ${ }^{1}$ \\ ${ }^{1}$ Siberian Federal University, Krasnoyarsk, Russia \\ ${ }^{2}$ Institute of Thermophysics SB RAS, Novosibirsk, Russia \\ ${ }^{3}$ Novosibirsk State University of Architecture and Civil Engineering, Russia
}

\section{E-mail:perpetuityrs@mail.ru}

The numerical investigation of the flow regimes in the T-shaped microchannels with different width-to-height aspect ratios of mixing channel was carried out. In the first case, the mixing channel width was varied from $200 \mu \mathrm{m}$ to $1000 \mu \mathrm{m}$ while its height was constant; in the second case, the mixing channel height was varied from $100 \mu \mathrm{m}$ to $2000 \mu \mathrm{m}$, while its width was constant. The Reynolds number was varied from 5 to 700 . The dependences of fluids mixing efficiency and the pressure drop on the Reynolds number at different width-to-height aspect ratios of mixing channel were numerically established. The correlations to determine the critical Reynolds number, as well as the friction factor at different widths and heights of the mixing channel, were proposed. The mixing efficiency, reduced to the pressure drop and the volume unit was analyzed for the first time. The optimal range of parameters in terms of the working efficiency of the micromixer was determined.

Keywords: Hydrodynamics, numerical simulation, T-shaped micromixer, microagitator, microreactor, mixing, width-to-height aspect ratio, engulfment regime, pressure drop, CFD.

\section{INTRODUCTION}

Micro- and nanotechnologies are rapidly developing at the present day. They are successfully used in various fields of science and industry. To date, the use of microreactor systems from the field of purely scientific interests and the creation of prototypes has been singled out as a separate branch of the modern production cycle. In particular, microfluidic (microreactor) technologies are successfully used for the synthesis of various organic and inorganic substances (especially high-purity), for the development of new dosage forms and diagnostic systems, as well as in biotechnological research of various kinds. Microtechnologies provide easier process control, higher performance with miniaturization of equipment and higher 
product quality compared with standard technologies. Microreactors are also used to transport the nanoparticles, bacteria, DNA molecules, for cooling microelectronic devices, as chemical reactors for microscopic amounts of matter, etc. Obtaining of micro- and nanoparticles of predetermined stable characteristics in microreactors cause the considerable interest in the pharmaceutical applications of microreactor technologies. Such particles can be further used both as components of new dosage forms and for creating diagnostic test systems. Control of pressure, temperature, reaction time and flow rates in small volume reactors is much easier and more efficiently. Hence, the main undeniable advantages of microsystems are the safety of carrying out highly exothermic reactions and working with toxic or explosive reagents, carrying out the reactions under supercritical conditions, and, in whole, the significantly reducing of the research costs, as well as implementation and scaling of chemical processes.

Many studies have noted that the use of microdevices allows significantly enhancing the physicochemical processes in comparison to classical space consuming reactors [1-3]. However, despite the obvious benefits of microchannel technology, there are a number of specific problems. Most microchannel devices that used in chemistry and biology require fast and effective mixing of substances [1-5]. At the same time, the flow in microchannels is predominantly laminar and mixing occurs through diffusion, i.e. very slowly. Therefore, the design and optimization of micromixers with the shortest mixing time is an actual problem in the development of microchannel devices.

The T-shaped micromixers (Fig.1) are most common among all possible forms of micromixers and microreactors. Such micromixers are an integral part of almost any complex micromixing equipment while using as an independent micromixer they show a quite good efficiency. There are many experimental and numerical investigations of flow and mixing regimes in such micromixers [6-27]. The studies of mixing of two fluids with the same thermophysical properties revealed six different flow regimes, which depend on the Reynolds number:

$\operatorname{Re}<5$ - steady stratified flow;

$5<\operatorname{Re}<145$ - steady symmetric vortex flow;

$145<\operatorname{Re}<240$ - steady asymmetric vortex flow;

$240<\operatorname{Re}<400-$ periodic flow;

$400<\operatorname{Re}<600$ - unsteady quasiperiodic flow;

$\operatorname{Re}>600$ - stochastic flow, laminar-turbulent transition.

Furthermore, the dramatic increase in mixing efficiency at a transition from symmetric mode to asymmetric one for stationary flow regime (called the engulfment regime) was noted in most research works. Such a transition occurs abruptly when the Reynolds number reaches some critical value $\mathrm{Re}_{c r}$. That regime is of the greatest interest for researchers because the 
mixing efficiency increases abruptly in this mode while the pressure drop in the mixing channel increases insignificantly. The value of the critical Reynolds number depends on many factors.

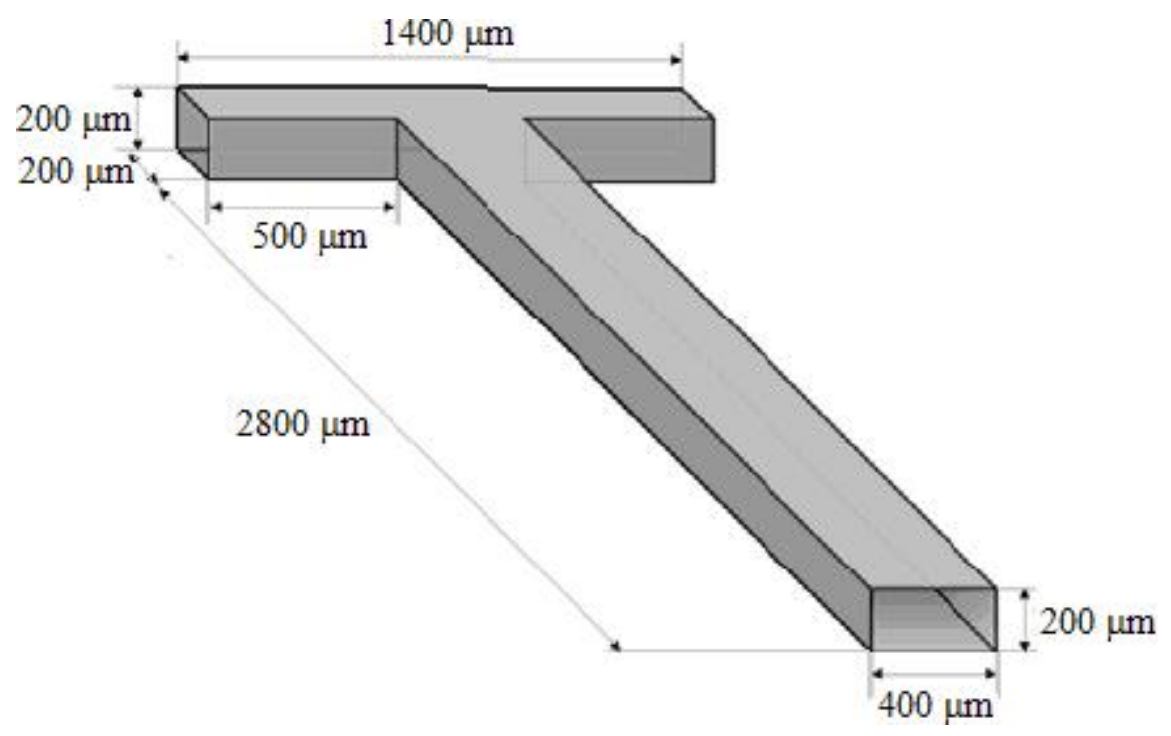

Figure 1. The geometric configuration of the task

It was shown in [14-22] that the value of the critical Reynolds number depends on the thermophysical properties of mixing fluids. Thus, it was found that as the fluids viscosities ratio increases the critical Reynolds number increases too, but such value doesn't depend on the fluids densities ratio. Also, the influence of the rheology of the mixing fluids on the shift of flow regimes in T-shaped micromixer [19, 21, 22] was pointed out.

Besides, it is well known, that the critical Reynolds number depends on the T-channel geometry [22, 24, 28-32]. The investigation of geometric dimensions effect on the mixing efficiency of T-shaped micromixers was carried out in several works. E.g., in [28] the Newtonian fluid mixing in micro T-mixer with variable inlets widths was experimentally characterized. It was shown that the use of asymmetrical T-micromixers leads to increase the quality of mixing, and as the degree of asymmetry is higher, the benefits are greater.

A detailed investigation of the influence of the geometric dimensions of the mixing channel on the value of the critical Reynolds number was carried out in [24]. The mixer dimensions were varied in wide ranges. A correlation to determine $\mathrm{Re}_{c r}$, that depends on the geometric dimensions of the mixer, was proposed as a result.

An investigation of the effects of aspect ratio and shear-thinning on the bifurcation in a T-channel junction was carried out in [22]. It was shown there that the change in flow conditions from symmetric to asymmetric flow is, in most cases, a steady supercritical pitchfork bifurcation. Besides, it was shown that the critical Reynolds number is different for T-shaped microchannels with different geometric dimensions. The obtained results were 
compared with dimensionless criteria from [24] and it was found that an existing correlation for predicting the critical conditions can fail under certain aspect ratio combinations. Also, the critical Reynolds number, obtained in [27], was twofold higher, then the value calculated using the correlation from [24]. The experimental and numerical investigation of the influence of the micromixer height on the onset of the engulfment regime was carried out in [32]. Several different geometrical configurations of T-shaped micromixer were investigated, and it was found that the correlation from [24] predicted $\mathrm{Re}_{c r}$ correctly in some cases, but underestimated that value sufficiently in some other cases. Besides, it was found that for some configurations the engulfment regime does not occur at all.

It should be noted in addition, that most of the huge amount of works, devoted to the study of micromixers, focus on the analysis of mixing efficiency, and only in some works [11, 13-16, 19, 27], there are some data on the magnitude of pressure loss in these mixers. In this regard, at the present time, there are practically no systematic data and correlations allow calculating the pressure drop in the T-shaped micromixers in a wide range of parameters. Pressure drop is quite an important value since it characterizes the energy consumption during the pumping of fluids, which is important for the practical application of mixers. Thus, the aim of the present work is to carry out systematic numerical investigations of mixing modes in T-shaped micromixers and to create the corresponding correlation dependences of the mixing efficiency and the pressure drop in T-shaped micromixers in wide ranges of their dimensions.

\section{MATHEMATICAL MODEL AND FORMULATION OF THE PROBLEM}

Incompressible flows of multi-component and generally non-Newtonian fluids, which are described using a hydrodynamic approach based on the solution of the Navier-Stokes equations were considered in this work. Currently, it was shown in numerous experiments that such description for fluids works well down to the channels sizes of about 1 micron.

In general, the Navier-Stokes equations system has the following form:

$$
\frac{\partial \rho}{\partial t}+\nabla(\rho \mathbf{v})=0 \quad \frac{\partial \rho \mathbf{v}}{\partial t}+\nabla(\rho \mathbf{v v})=-\nabla p+\nabla \mathbf{T}
$$

Here $\rho$ is the fluid density, $p$ is the pressure, $\mathbf{v}$ is the velocity, and $\mathbf{T}$ is the tensor of viscous stresses, which components are determined as:

$$
\mathbf{T}_{i j}=\mu \cdot\left(\frac{\partial u_{j}}{\partial x_{i}}+\frac{\partial u_{i}}{\partial x_{j}}\right)
$$

Here $\mu$ is the mixture viscosity and $u_{i, j}$ are the velocity vector components.

The effective viscosity and the density of the mixture are determined through the mass 
fraction of its components $f$ and effective viscosities $\mu_{1,2}$ and partial densities $\rho_{1,2}$ of pure components, respectively:

$$
\mu=f \mu+(1-f) \mu_{2} \quad \frac{1}{\rho}={ }^{f_{+}} \frac{(1-f)}{\rho_{1}} \rho_{2}
$$

Here the evolution of mass concentrations is determined by the equation:

$$
\frac{\partial \rho f}{\partial t}+\nabla(\rho f \mathbf{v})=\nabla(\rho D \nabla f)
$$

where $D$ is the diffusion coefficient.

The software package for computational fluid dynamics SigmaFlow was used to solve the equations system described above. A detailed description of the numerical algorithm of this software tool is given in [33]. Here the main points of numerical technique will be noted. The difference analogue of the convective-diffusion equations (1) and (2) is determined using the finite volume method [34-36] for structured multi-block grids. In this case, the resulting scheme is automatically conservative. The method consists of splitting the computational domain into control volumes and integrating the original conservation equations for each control volume to obtain finite-difference equations. Approximation of convective terms of transfer equations (1) and (2) is carried out using the second order upwind QUICK [37] and TVD [36] schemes, respectively. To approximate the unsteady terms of hydrodynamics equations, the second order implicit scheme was applied. Diffusion fluxes and source terms were approximated by finite-volume analogues of the central-difference correlations with the second order of accuracy. The connection between the velocity and pressure fields, ensuring the fulfilment of the continuity equation, was implemented by means of the SIMPLEC procedure for combined grids [34]. Rhi-Chow approach consisting of the introduction of monotonizator into the equation (1) for the pressure correction [38] was used to eliminate the pressure field oscillations. The difference equations obtained after discretization of the original system of differential equations were solved by an iterative method with the use of the algebraic multigrid solver [39].

The four-block grid of 9.5 million grid nodes was used for calculations in this work. Preliminary calculations have shown that such level of grid particularization is acceptable in terms of calculation accuracy. The constant fluid flow rate with a steady-state velocity profile was set at the inlets of micromixer. Neumann conditions meaning zero derivatives of all scalar quantities normal to the outlet surface were set at the outlet of the mixing channel. The walls of the mixer were considered as insulated. The no-slip condition was taken as a boundary condition on the channels walls for the velocity vector components. The applicability of such 
type of boundary conditions till the channels sizes up to $50 \mu \mathrm{m}$ has been shown in $[1,12,13]$.

The base configuration channel dimensions are shown in Fig. 1. The channel thickness is $200 \mu \mathrm{m}$, while the width of its narrow and wide parts is $200 \mu \mathrm{m}$ and $400 \mu \mathrm{m}$, respectively. Pure water was supplied to the mixer through one of the inlets with the mass flow rate $Q_{i n}$. Tinted water was supplied through another inlet with the similar flow rate. The density of both fluids was $1000 \mathrm{~kg} / \mathrm{m}^{3}$, the viscosity was $0.001 \mathrm{~Pa} \cdot \mathrm{s}$, the diffusion coefficient was $D=2.63 \times 10^{-10} \mathrm{~m}^{2} / \mathrm{s}$. The change in the flow regimes in the microchannel is characterized by the Reynolds number, defined as follows:

$$
\operatorname{Re}=\frac{Q d_{h}}{\mu w h}
$$

where $Q=2 Q_{i n}$ is the mean mass flow rate in the mixing channel, $w, h$ are the mixing channel width and height, $d_{h}$ is the hydraulic diameter.

The pressure drop between one of the mixer inlets and the outlet, as well as mixing efficiency, were determined in this study. The mixing efficiency is usually quantified using the parameter $M=1-\left(\sigma / \sigma_{0}\right)^{0.5}[6-11,13-21,27-29,31]$, where $\quad \sigma=V^{-1} \int_{V}(f-\langle f\rangle)^{2} d V \quad$ is the root-mean-square deviation of the mass fraction of mixture component $f$ from its average value $\langle f\rangle, \quad \sigma_{0}=\langle f\rangle(1-\langle f\rangle)$ is the maximum root-mean-square deviation, and $V$ is the volume of the computational domain.

Let us briefly describe the change of flow regimes for the basic geometry of the mixer.

The Reynolds number of about 1 corresponds to creeping stratified flow accompanied by a weak mixing. The increase in Reynolds number in the mixer leads to the development of a pair of symmetrical horseshoe vortices, which are formed near the left end wall of the mixer and propagate into the mixing channel (Fig. 2a). This is a so-called steady symmetric vortex flow regime. The vortices structures in such a regime are shown in Fig. 2a through $\lambda_{2}$ isosurfaces that is the second eigenvalue of the tensor $\left(\mathbf{S}^{2}+\boldsymbol{\Omega}^{2}\right)$, where $\mathbf{S}$ is the strain rate tensor and $\Omega$ is the vorticity tensor. As one can see in that figure, the flow in such case is symmetrical relative to the central longitudinal plane of the mixer. Each such horseshoe vortex located in the borders of corresponding fluids and didn't cross the interface between miscible fluids. Therefore, the interface between two media remains almost flat (see Fig. 3a). 

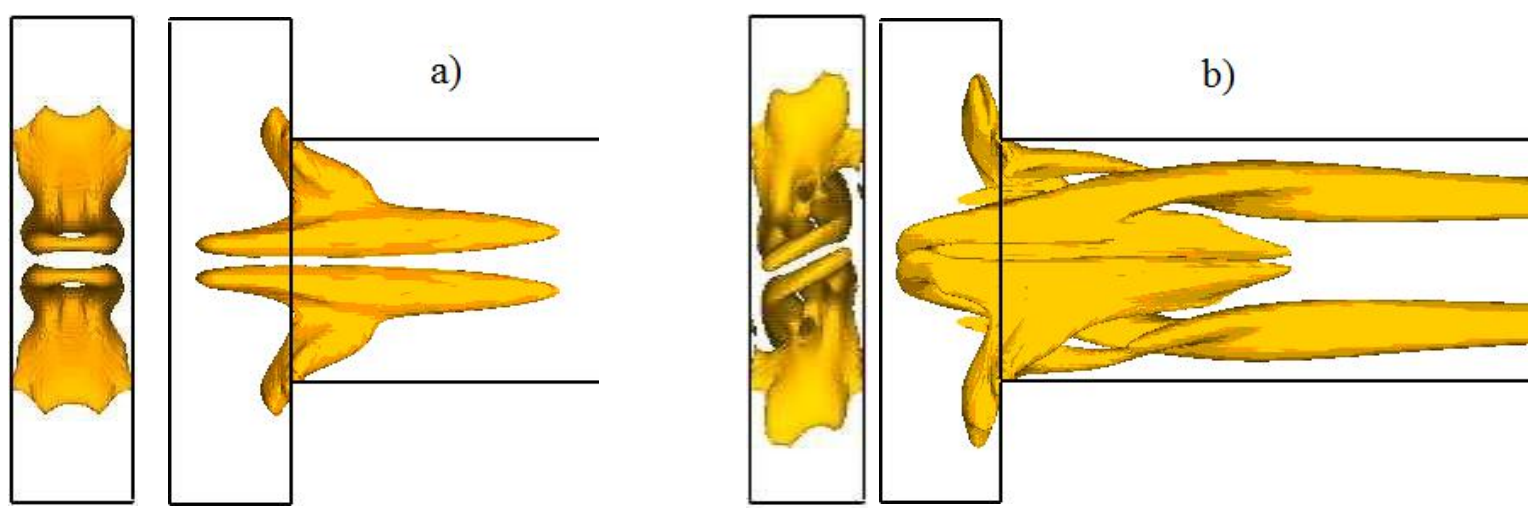

Figure 2. $\lambda_{2}$ isosurfaces: $\operatorname{Re}=140(\mathrm{a}) ; \operatorname{Re}=150(\mathrm{~b})$. Left parts correspond to mixer end-view; right parts correspond to side-view

When the Reynolds number further increases and reaches the critical value, the flow structure in the mixer changes abruptly. A pair of horseshoe vortices turn on an angle of $30^{\circ}$ in relation to the central longitudinal plane of the mixer due to the development of the TaylorGertler instability, which caused by quarter-turn of each fluid (see Fig. 2b). This is a so-called steady asymmetric vortex flow regime. The critical Reynolds number for basic configuration was $\operatorname{Re}_{c r}=145$. The rearranging of the flow in the mixer channel leads to the formation of the layered S-shape structure of miscible fluids (see Fig. 3b). The interface of miscible fluids in such a layered structure is very well developed that leads to a sharp increase in the mixing efficiency. When flow changing from the symmetric flow regime $(\operatorname{Re}<145)$ to the engulfment flow regime $(\operatorname{Re}>145)$ the mixing efficiency increases more than 45 times.
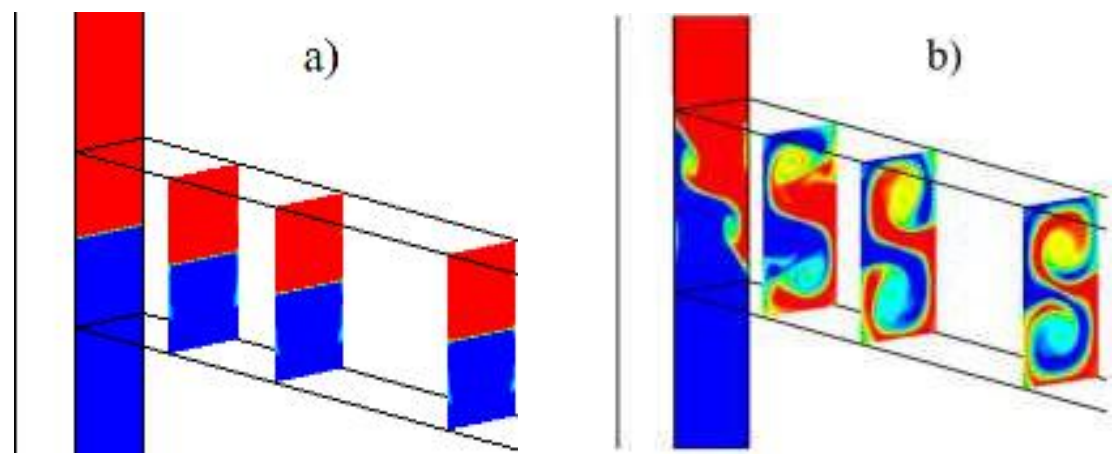

Figure 3. Concentration isolines in different cross-sections of the mixer:

$$
\operatorname{Re}=140(a) ; \operatorname{Re}=150(b)
$$

\section{RESULTS OF NUMERICAL SIMULATION}

Investigation of the mixing channel width effect. In the first series of calculations, the width of the mixing channel of the T-shaped microchannel was varied. The microchannel with a height of $h_{0}=200 \mu \mathrm{m}$ and a width of $w_{0}=400 \mu \mathrm{m}$ shown in Fig. 1 was the basic 
configuration. The remaining configurations were obtained by multiplying its width by the corresponding coefficients. All other dimensions of the microchannel remained unchanged. The micromixers with widths of mixing channel from $0.5 w_{0}$ to $5 w_{0}$ were considered. The hydraulic diameters of the narrowest and the widest mixing channels were equal to $200 \mu \mathrm{m}$ and $363.34 \mu \mathrm{m}$, respectively.

As a result of the calculations, a number of similarities and differences were revealed in the mixing of two fluids in T-shaped microchannels of different widths. First of all, this relates to the engulfment flow regime. In particular, it was found that such a flow regime is observed for each considered configurations. It can be clearly seen in Fig. 4, which represents the isolines of the concentration at the outlet of the mixing channel for different widths of that channel after the onset of the engulfment regime. However, it can be seen from that figure that the shape of the interface between two media in this mode differs significantly for different configurations. For example, the S-shaped interface shown for the basic configuration is practically not observed for the channel with the width of $0.5 w_{0}$. When the width increases, the concentration isolines profile also stretching and for the $1.75 w_{0}$ channel additional swirl flow regions appear. For the channel with the width of $2 w_{0}$, they become strongly pronounced. As the channel width further increases, such structure disappears. In addition, as the Reynolds number further increases, an unsteady periodic flow regime occurs for each of the considered configurations. The dependences of the mixing efficiency on the Reynolds number shown in Fig. 5a, also confirm the presence of the engulfment regime for each of the configurations (that value increases abruptly in the onset of such regime). A strict dependence of the critical Reynolds number on the width of the mixing channel can be observed in that figure. It can be seen, that as the channel width increases, that value shifts toward smaller Reynolds numbers, and, at the same time, the mixing efficiency in the engulfment regime increases too. This is due to the increase in the contact area of two miscible fluids (see Fig. 4).

It should be noted, that when $\mathrm{Re}<\mathrm{Re}_{c r}$ (symmetric regime), the mixing efficiency almost doesn't depend on the width of the mixing channel. It can be explained by that the contact area of two miscible fluids remains almost flat. So, as the height of the mixing channel doesn't change, the contact area of two miscible fluids doesn't change too. The dependence of the pressure drop in the mixing channel, shown in Fig. 5b also shows a strict correspondence between that value and the width of the mixing channel. In this case, the pressure difference decreases with increasing channel width, which is natural, since the crosssectional area of the mixing channel increases. 


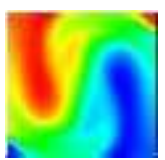

a)

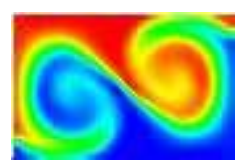

b)

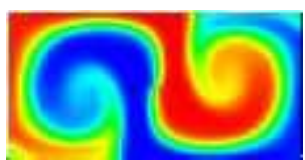

c)

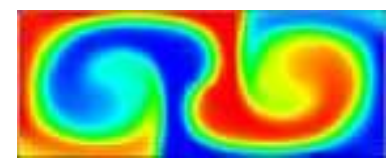

d)

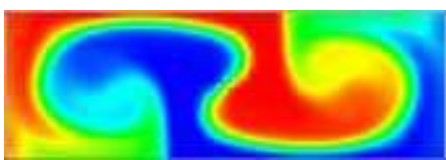

e)

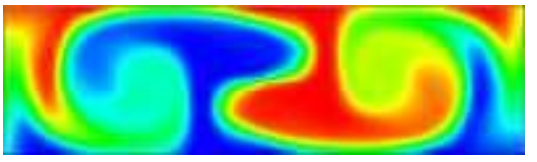

f)

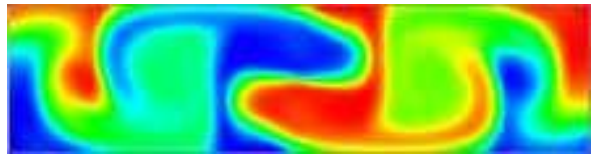

g)

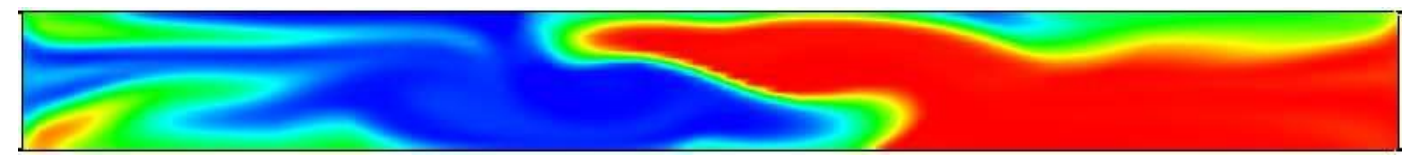

h)

Figure 4. The concentration isolines at the outlet of the mixing channel, $w / w_{0}: 0,5$ (a); 0,75 (b); 1 (c); 1,25 (d); 1,5 (e); 1,75 (f); 2 (g); 5 (h)

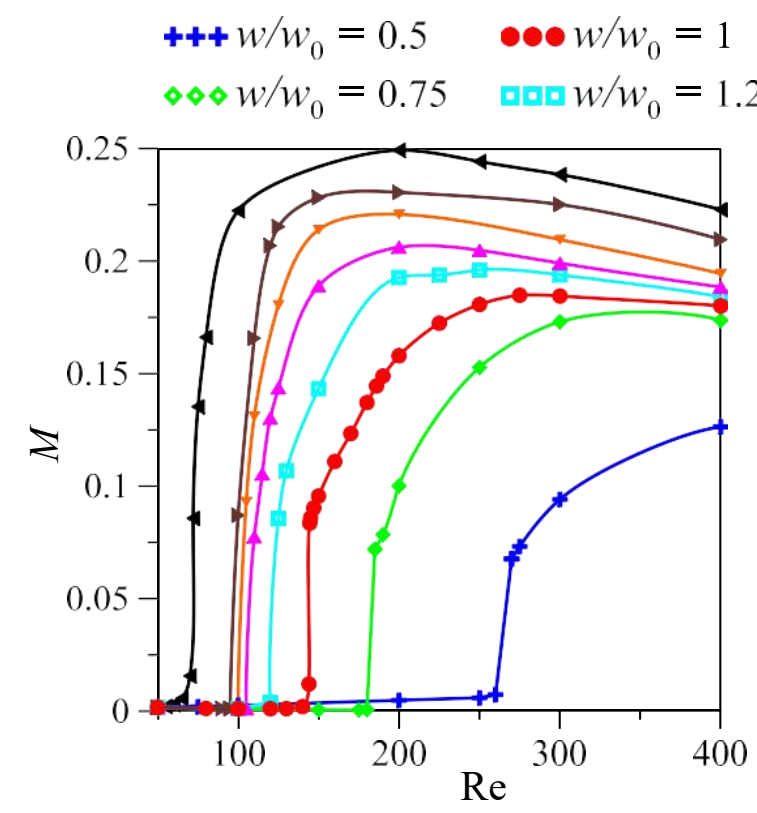

a)

$$
\begin{array}{ll}
\Delta \Delta \Delta w / w_{0}=1.5 & \mapsto w / w_{0}=2 \\
\nabla \nabla \nabla w / w_{0}=1.75 & \hookrightarrow \triangleleft \triangleleft w / w_{0}=5
\end{array}
$$

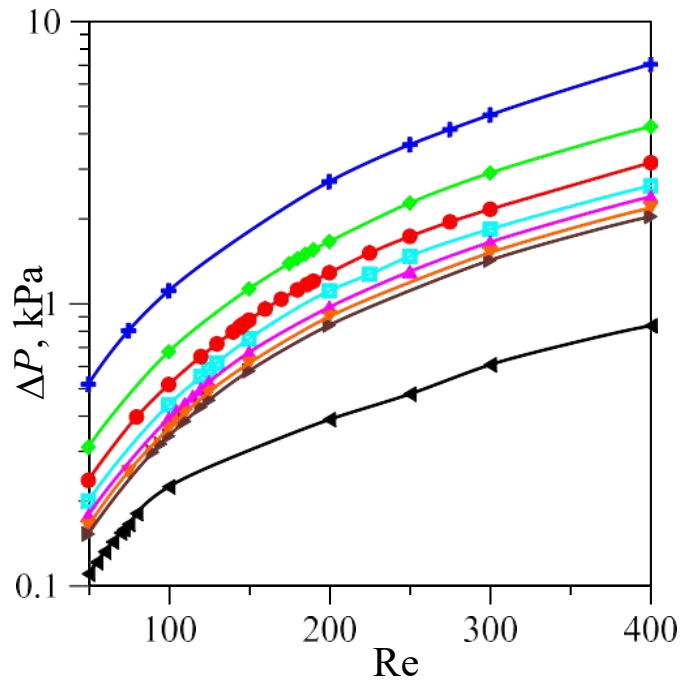

b)

Figure 5. The dependences of mixing efficiency (a) and pressure drop (b) in the mixing channel on Reynolds number for mixers with different widths

An analysis of the flow vortex structure in the mixers shows, that in the range of the dimensionless channel width $w / w_{0}$ from 0.75 to 2 that structure is similar to that for the basic mixer configuration (see Fig. 2). A special feature of the flow structure was found in the microchannel with a square cross-section of the mixing channel $\left(w / w_{0}=0.5\right)$. The vortices structures for such configuration at different Reynolds numbers are shown in Fig. 6. It was found, that the pair of horseshoe vortices also forms in the steady symmetric flow regime in 
such configuration of the T-shaped microchannel (Fig. 6a), and in the engulfment flow regime these vortices merge and form a single-vortex structure (Fig. 6b). This is due to an insufficiency of space for further expansion of two vortices.

Another interesting mode is realized in configurations with high values of the dimensionless width of the mixing channel $w / w_{0}>2$. As can be seen in Figs. 4g, 7, in such mixers appear additional vortices, which are another pair of Dean vortices but are in an embryonic state. A similar cellular structure of several Dean vortices was observed in [40], where the influence of various parameters on the origin and behaviour of Dean vortices was studied. The presence of the additional vortices, that appear when the mixing channel is expanded, leads to an extra increase in the mixing efficiency for such micromixers configurations (see Fig. 5a). As one can see, in the region of $\operatorname{Re}>\operatorname{Re}_{c r}$, as the dimensionless width of the mixing channel $w / w_{0}$ increases, the mixing efficiency increases too.
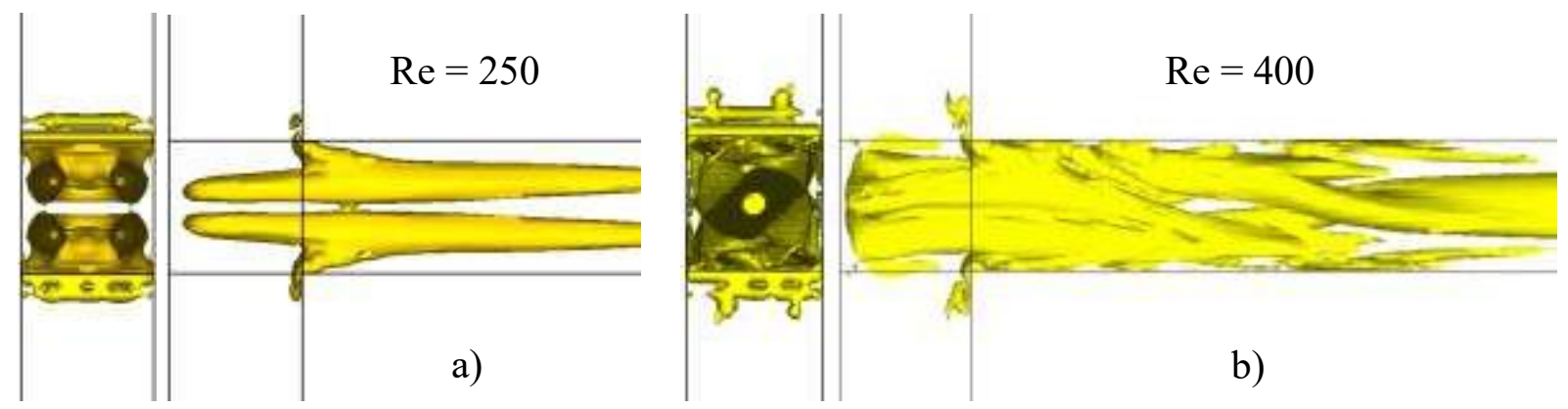

Figure 6. $\lambda_{2}$ isosurfaces, front and side views, $w / w_{0}=0.5$
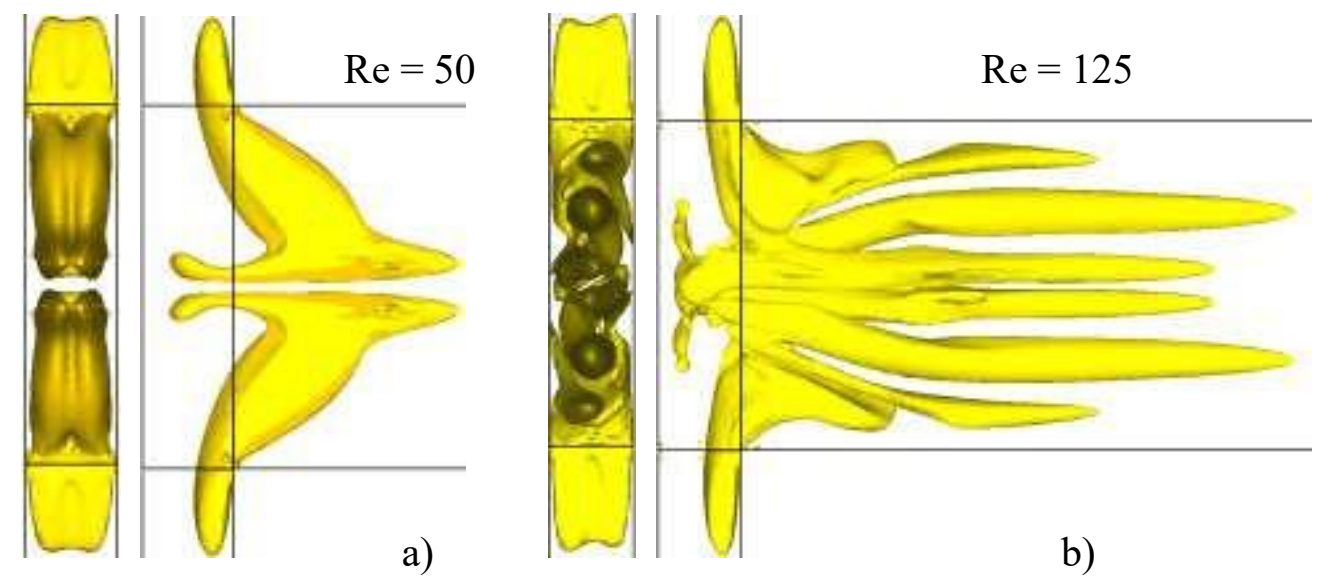

Figure 7. $\lambda_{2}$ isosurfaces, front and side views, $w / w_{0}=2$

Investigation of the mixing channel height effect. In the second series of calculations, the height of the mixing channel of the T-shaped microchannel was varied. The microchannel with the height of $h_{0}=200 \mu \mathrm{m}$ and the width of $w_{0}=400 \mu \mathrm{m}$ shown in Fig. 1 
still was the basic configuration. The remaining configurations were obtained by multiplying its height by the corresponding coefficients. All other dimensions of the microchannel remained unchanged. The micromixers with heights of mixing channel from $0.5 h_{0}$ to $10 h_{0}$ were considered. The hydraulic diameters of the lowest and the highest mixing channels were equal to $160 \mu \mathrm{m}$ and $666.67 \mu \mathrm{m}$, respectively.

The results of this calculation series significantly differ from the previous one. In particular, the engulfment flow regime is observed only for configurations with mixing channel heights from $0.75 h_{0}$ to $1,5 h_{0}$. It can be clearly seen in Fig. 8, which represents the isolines of the concentration at the outlet of the mixing channel for different widths of that channel after the onset of the engulfment regime. Similar conclusions can be obtained from the analysis of Fig. 9a, which represents the dependence of the mixing efficiency on the Reynolds number for different micromixers configurations. It can be seen, that there is a dependence of the mixing efficiency on the mixing channel height in the region before the transition to the engulfment regime $\left(\operatorname{Re}<\operatorname{Re}_{c r}\right.$ ). It was shown, that as the mixing channel height increases, the mixing efficiency in that region also increases. It can be explained in such a way as the contact area of two miscible fluids increases with increase in height of the mixing channel. It becomes clear from the comparison of the Figs. 8a and 8f. The analysis of the mixing efficiency in the engulfment regime $\left(\operatorname{Re}>\operatorname{Re}_{c r}\right)$ showed, that that value increases with increase in the dimensionless height of the mixing channel $h / h_{0}$. It can be also explained by the presence of the more developed fluids contact area in the higher channels.

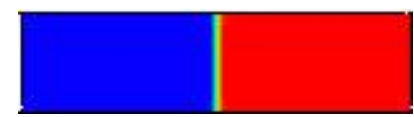

a)

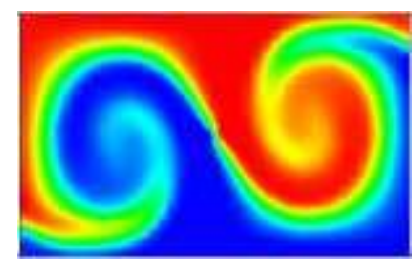

d)

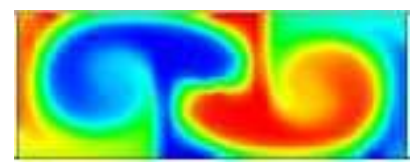

b)

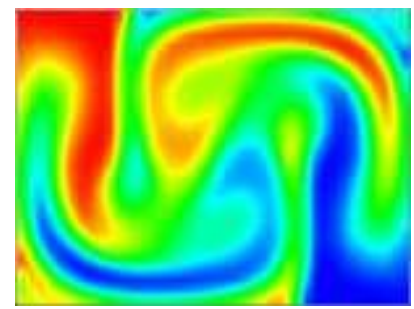

e)

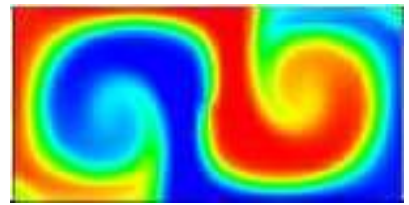

c)

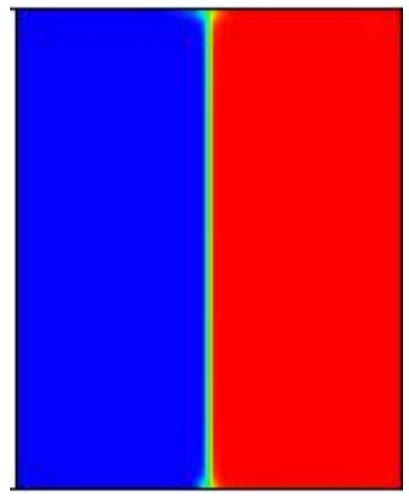

f)

Figure 8 . The concentration isolines at the outlet of the mixing channel, $h / h_{0}: 0.5$ (a); 0.75 (b); 1 (c); 1.25 (d); 1.5 (e); 2.5 (f) 


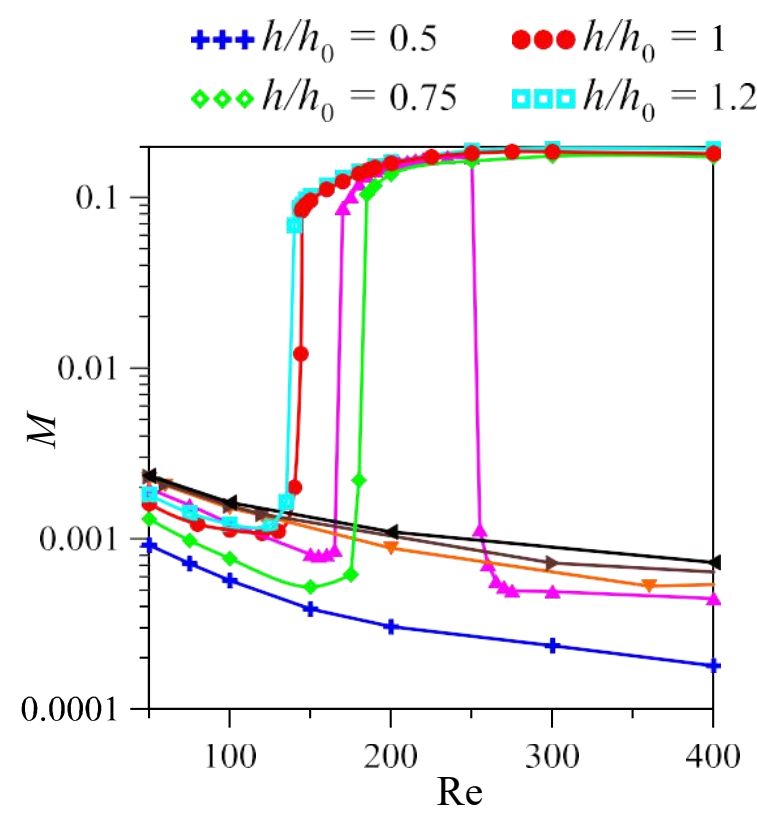

a)

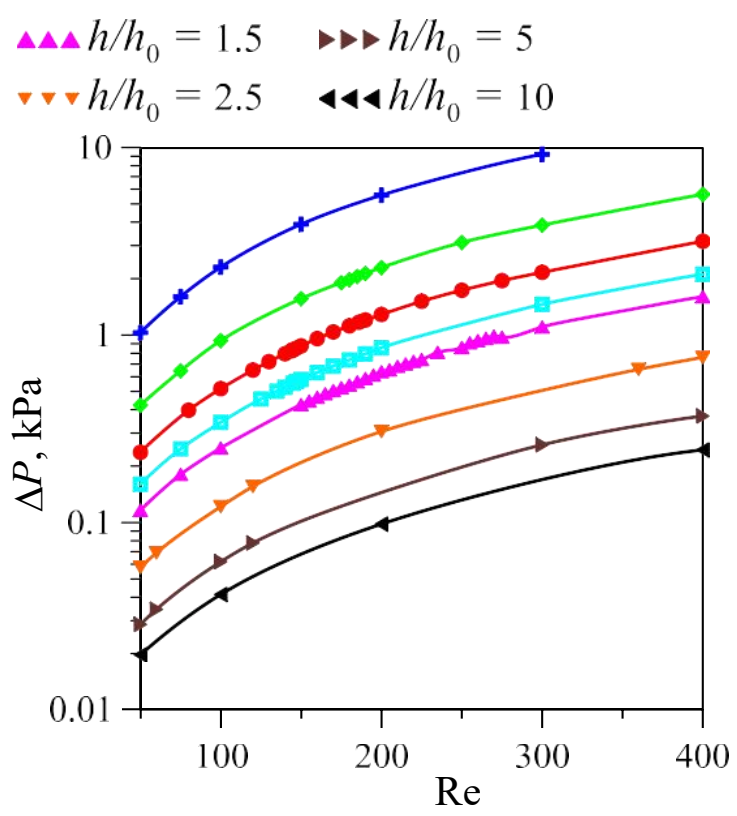

b)

Figure 9. The dependences of mixing efficiency (a) and pressure drop (b) in the mixing channel on Reynolds number for mixers with different heights

The evolution of the vortex structure of the flow with increasing in dimensionless channel height is shown in Figs. 10, 11. Since the range of the dimensionless height of the mixing channel for the engulfment regime is rather narrow $\left(0.75<h / h_{0}<1.5\right)$, the vortex flow structure in that range is qualitatively similar to that observed for the basic configuration of the mixer.

It was found, that for the configurations with the height of the mixing channel greater than $1,5 h_{0}$ the onset of the engulfment regime is not observed. The unsteady periodic flow regime follows immediately after the steady symmetric vortex flow regime in such configurations. This is illustrated in Fig. 12. It can be seen that the pair of symmetric horseshoe Dean vortices, typical for the symmetric vortex flow regime, is formed ever for very high configurations. Herewith, it was found that each branch of such horseshoe vortex forms near upper and lower walls of the mixing channel, respectively (see Fig. 12a). So, as the height of the mixing channel increases, the distance between each branch of each horseshoe vortex increases too. As a result, the vortices do not interact with each other, and the engulfment regime does not appear even at high Reynolds numbers up to the onset of turbulence (see Fig. 12c). Thus, it was shown that the key factor in the transition from symmetric to asymmetric flow regime is the distance between the vortices formed at the upper and lower walls of the mixing channel. Therefore, when the width of the mixing channel was varied, the engulfment regime was observed for each of the considered configurations, 
because the distance between the vortices remained unchanged. And when the height of the mixing channel is varied, the engulfment flow regime is observed only in those channels, in which the distance between the vortices, on the one hand, is greater than necessary for the existence of two vortices and, on the other hand, is sufficient for their interaction.

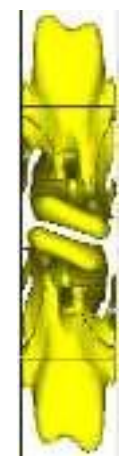

a)

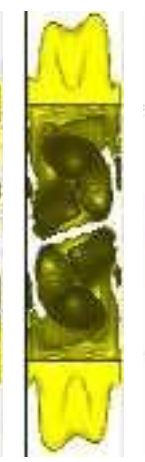

b)

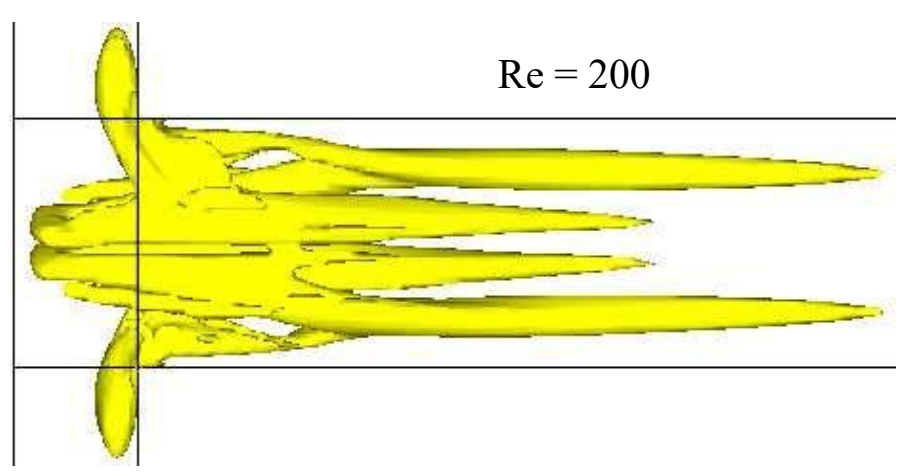

c)

Figure 10. $\lambda_{2}$ isosurfaces, back, front and side views, $h / h_{0}=0,75$

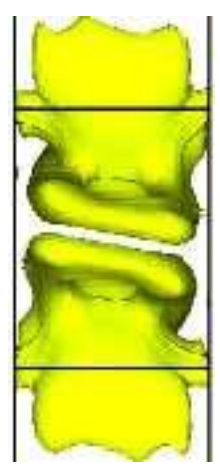

a)

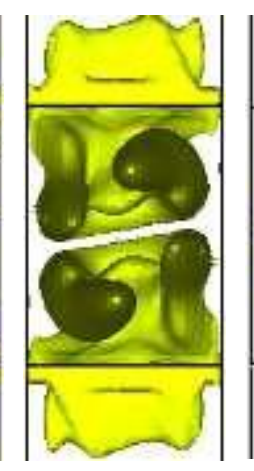

b)

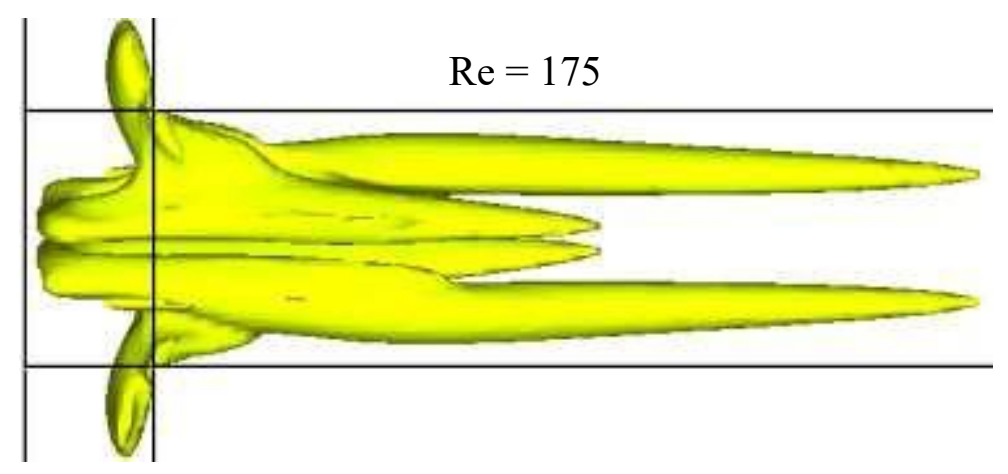

c)

Figure 11. $\lambda_{2}$ isosurfaces, back, front and side views, $h / h_{0}=1,5$

Let us separately consider a micromixer with a width of $w_{0}$ and a height of $1.5 h_{0}$. The flow and mixing regimes in such configuration are significantly different from the all other configurations. In particular, it was found, that in such micromixer the steady symmetrical vortex flow regime is observed initially, after that at the Reynolds number of about 170 the engulfment flow regime occurs and exists till the Reynolds number of about 250, and then the flow returns to the previous regime and again becomes the steady symmetrical vortex. This is most clearly seen from the dependence of the mixing efficiency on the Reynolds number. It is seen that at $\operatorname{Re} \sim 170$ the mixing efficiency sharply increases, and at $\operatorname{Re} \sim 250$ it sharply decreases. Herewith, that effect is fairly stable and is retained on the sequence of the calculated grids and when the Reynolds number is changed with a very small step. Similar behaviour of the flow regimes was observed in [27], where the width-to-height ratio of the 
mixing channel was equal 0.75 , which identically the configuration with the width of $w_{0}=400 \mu \mathrm{m}$ and the height of $1,5 h_{0}=300 \mu \mathrm{m}$, considered here.

The dependence of the pressure drop in the mixing channel, shown in Fig. 9b, similar to the previous series of calculations shows a strict correspondence between this value and the height of the mixing channel. In this case, the pressure difference decreases with increasing channel height since the cross-sectional area of the mixing channel increases.

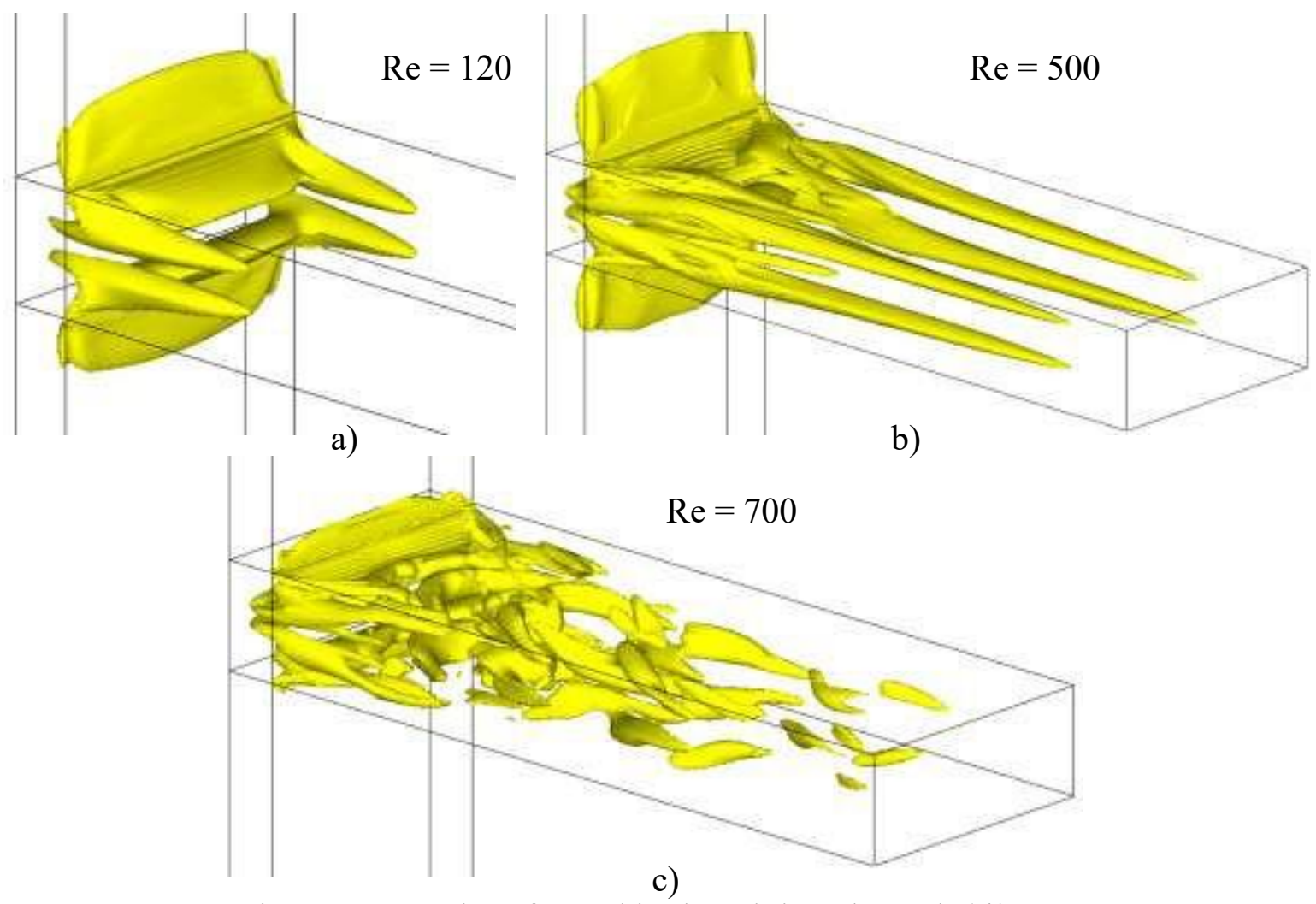

Figure 12. Vortices formed in the mixing channel, $h / h_{0}=5$

\section{Creating the correlation dependencies of pressure drop and critical Reynolds}

number. As already mentioned in the Introduction, the following correlation is proposed to determine the critical Reynolds number at which the transition to the engulfment flow regime occurs [24]:

$$
K=\operatorname{Re}^{0,82}\left(\frac{B}{\bar{C}}\right)^{-0,79}\left(\frac{D h_{i n}}{D h}\right)^{-1,5}\left(\frac{A}{C}\right)^{0,15}
$$

where $A$ is the width of the mixing channel, $B$ is the width of the inlet channel, $C$ is the height of the micromixer, $D h$ is the hydraulic diameter of the mixing channel, $D h_{i n}$ is the hydraulic diameter of the inlet channel. That correlation is chosen in such a way that the critical Reynolds number corresponds to the coefficient $K=100$. In paper [24] it has been alleged 
that such correlation is applicable over a wide range of geometric dimensions of the T-shaped microchannel: $A=60-1600 \mu \mathrm{m}, B=50-500 \mu \mathrm{m}, C=50-450 \mu \mathrm{m}$.

However, analyzing the results of fluids mixing, based on which that correlation was created, it can be seen that the applicable limits of geometric dimensions are wide only in absolute values, while in relative values (the width-to-height ratio of the mixing channel $w / h$ ) the sizes of microchannels vary within rather narrow limits. Therefore, it was shown in [22, $27,32]$ that that formula does not always correctly predict the critical Reynolds number. A comparison of the values of the critical Reynolds number obtained in our work with the correlation (4) is shown in Fig. 13. It can be seen from this figure that if the width of the mixing channel is varied, the correlation from [24] agrees well with our results up to a channel size of $1.5 w_{0}$, and, in the following, the discrepancies appear and increase as the width of the mixing channel increases (Fig. 13a). If the height of the mixing channel is varied, the correlation (4) agrees well with our results only within the dimensions of the mixing channel from $0.75 h_{0}$ to $1 h_{0}$ (see Fig. 13b). For higher values of the dimensionless channel height, not only quantitative but also qualitative differences in the results of calculations and correlations from work [24] are observed. As can be seen, the correlation (4) shows a monotonic decrease in the critical Reynolds number with an increase in the dimensionless height of the channel. At the same time, it was shown in the previous paragraph that the certain mixing channel heights, for which the engulfment flow regime exists, are required: the distance between the vortices must be, on the one hand, greater than necessary for the existence of two vortices and, on the other hand, sufficient for their interaction. This explains the extremum character of the dependence of $\operatorname{Re}_{c r}$ on $h / h_{0}$ (see Fig. 13b).

In this connection, the following correlation to determine the critical value of the Reynolds number is proposed in our work:

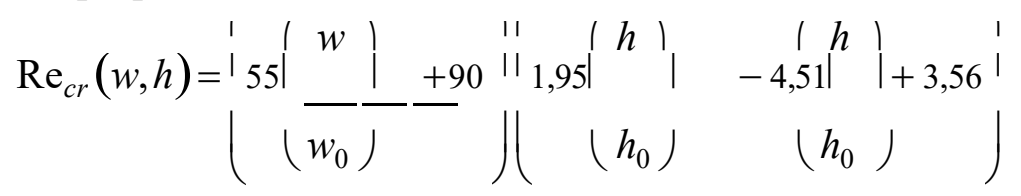

where $w, h$ are the width and height of the mixing channel for the current configuration, $w_{0}, h_{0}$ are the width and height of the mixing channel for the basic configuration. This correlation well describes the results obtained within $0.5 \leq w / w_{0} \leq 5 ; 0.75 \leq h / h_{0} \leq 1.5$. A comparison of this correlation and the obtained results are also shown in Fig. 13.

In addition to the critical Reynolds number, a correlation to determine the pressure drop in the T-shaped micromixer over a wide range of geometric dimensions of this channel was obtained. As already mentioned above, the most attention is paid to the efficiency of mixing in the investigations of the micromixers, and the pressure drop is taken into account 
rather rare, although this quantity is no less important. In order to determine the behaviour of the dependence of the pressure drop on the geometric dimensions of the mixing channel, the friction factor of the mixing channel was calculated, which was defined as:

$$
\lambda=\frac{2 \Delta P d_{h}}{\rho u^{2} L}=\frac{2 \Delta{ }_{h} P d^{3} \rho}{\operatorname{Re}^{2} \mu^{2} L},
$$

where $\Delta P$ is the pressure drop in the channel, $L$ is the channel length, and $\mathrm{U}$ is the average velocity in the mixing channel.

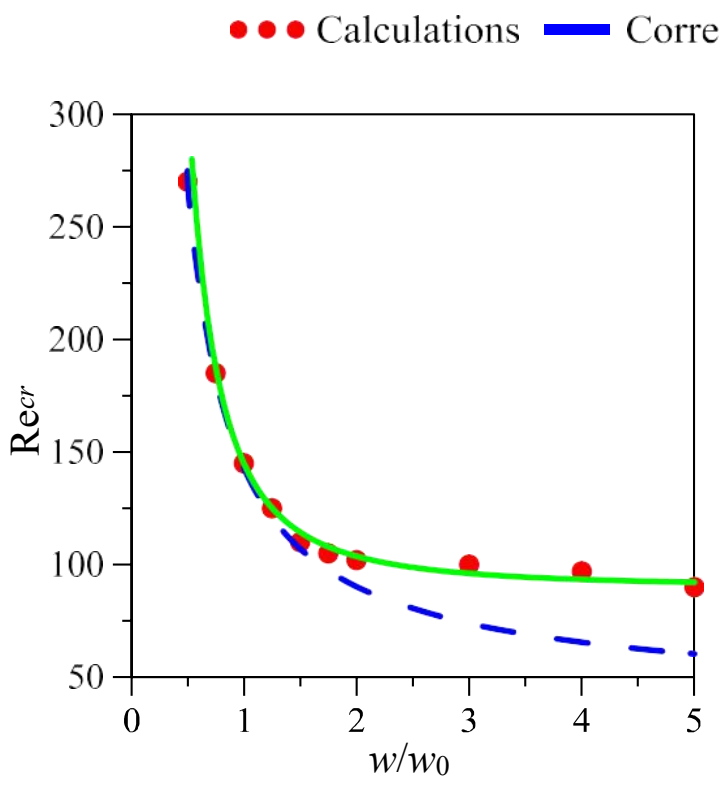

a)

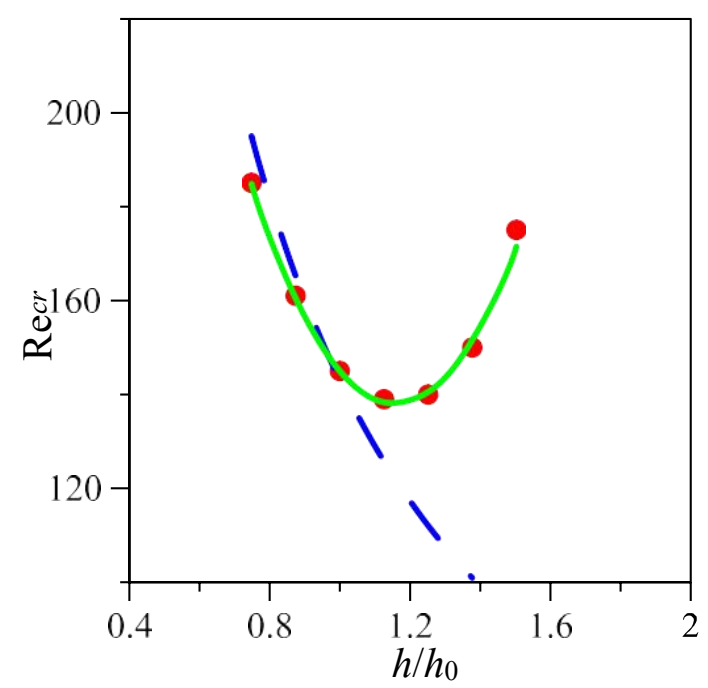

b)

Figure 13. The critical Reynolds number for T-mixers with different geometrical dimensions

It was found that the friction factor depends both on the Reynolds number and on the geometrical dimensions of the mixing channel. The analysis showed that the form of friction factor dependence on the Reynolds number for $\mathrm{Re}<100$ differs from the curve of that dependence for $\operatorname{Re}>100$. This can be explained by the fact that for $\operatorname{Re} \sim 100$ the formation of Dean vortices in the mixing channel begins for all configurations. As a result, the following correlation to determine the dependence of friction factor on the Reynolds number and the geometrical dimensions of the T-shaped mixer was obtained:

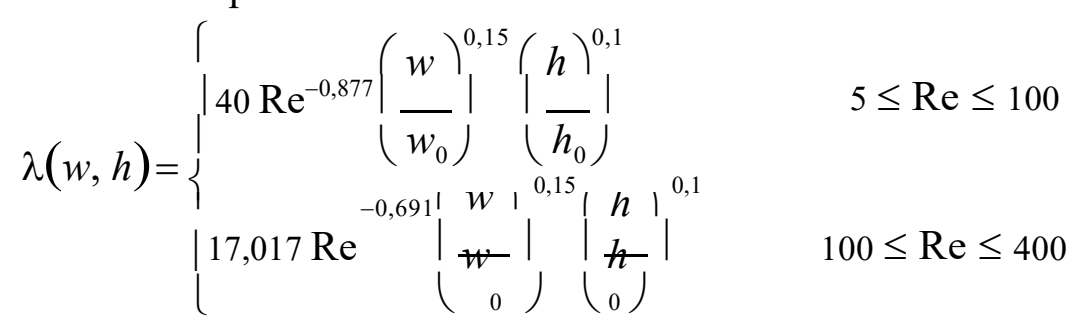

Comparisons of that correlation with the calculated results are shown in Fig. 14, 15, which represent the dependences of the friction factor on the Reynolds number, the 
dimensionless width and height of the mixing channel. Using that correlation, the pressure drop was obtained for all cases considered in the calculations. Comparison of the results showed that the difference between the calculation and the correlation does not exceed 5\%. These results are shown in Figure 16.

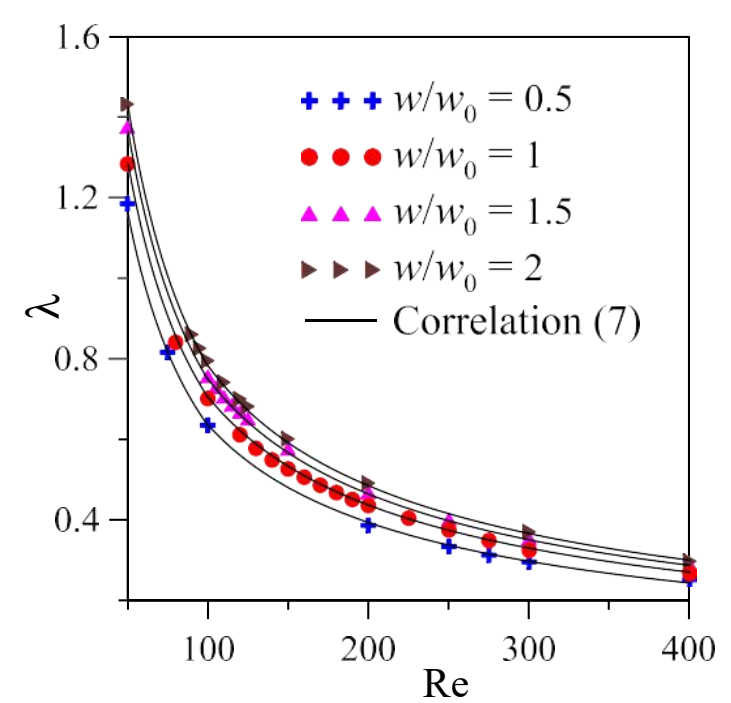

a)

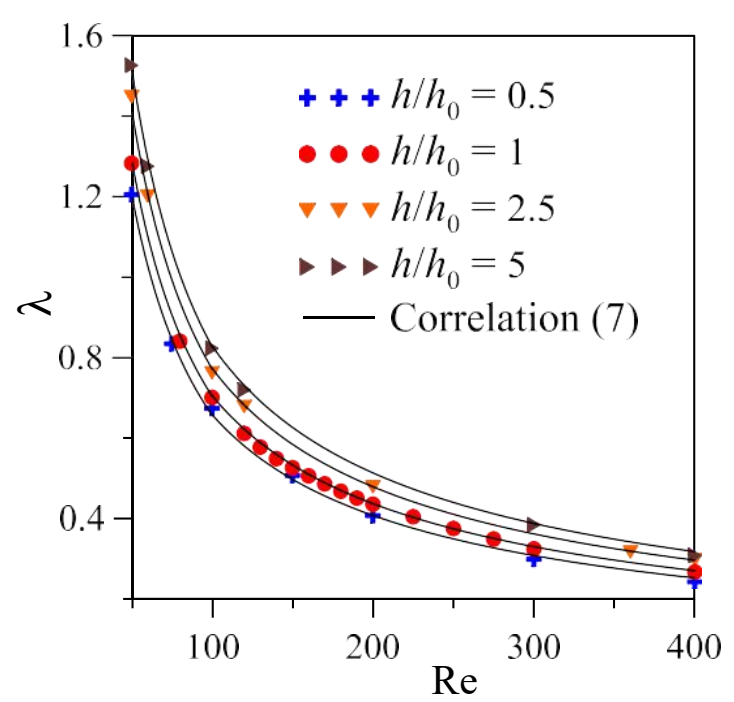

b)

Figure 14. The dependence of the friction factor on the Reynolds number for different configurations of the micromixer

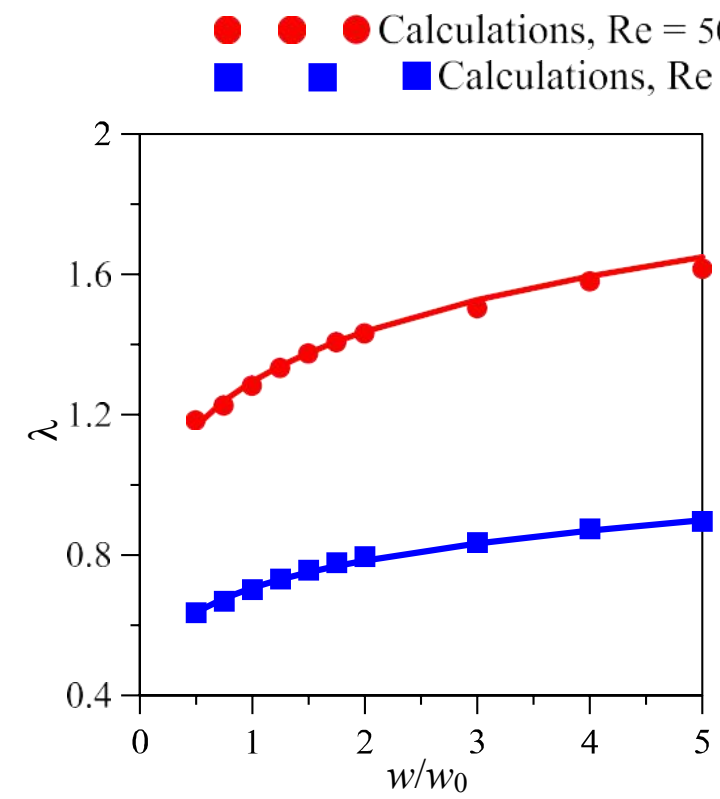

a)

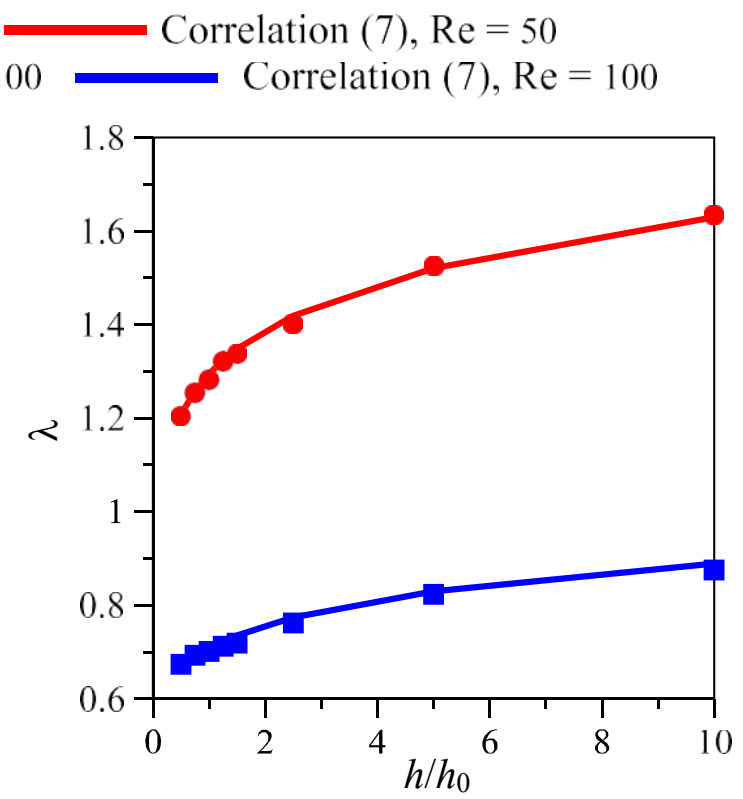

b)

Figure 15. The dependence of the friction factor on the geometrical configurations of the micromixer for different Reynolds numbers 


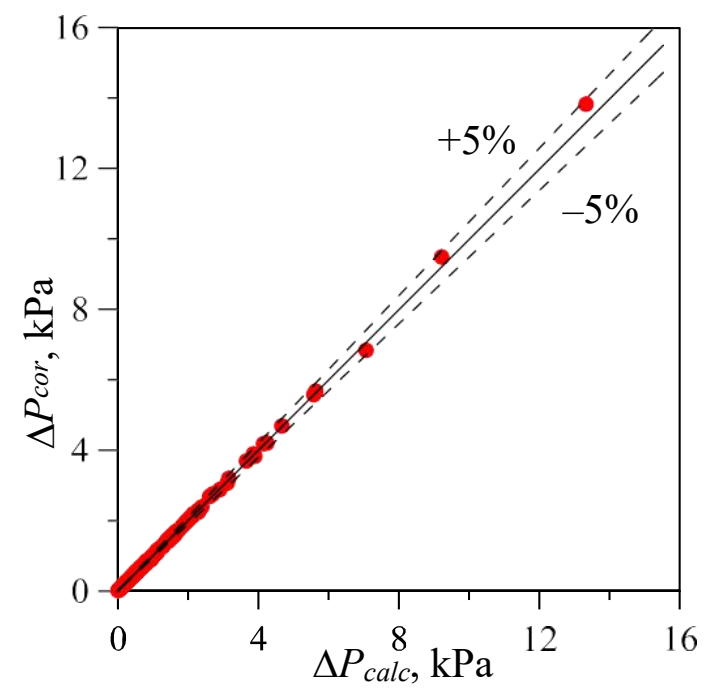

Figure 16. Comparison of the pressure drop in the mixing channel, obtained as a result of numerical simulation and using correlation (7)

On the optimal operating modes of $\mathbf{T}$-shaped micromixers. For the purpose to obtain the optimal mixing mode, it is necessary to consider not only the mixing efficiency in the mixer but also the energy that expends for pumping the fluid through this mixer. Those mixers and those regimes will be effective, in which the increase in the mixing efficiency is higher than the increase in the pressure drop under the same conditions. So to analyze the mixing efficiency in micromixers of different sizes, it is suggested to consider the reduced mixing efficiency. This value is defined as the ratio of mixing efficiency in the mixer to the volume of that mixer and to the magnitude of the pressure drop in it. The dependence of the reduced mixing efficiency on the Reynolds number for channels with different geometrical dimensions is shown in Fig. 17. It can be seen in that figure that for all considered micromixers, the reduced mixing efficiency has a maximum in the region of the Reynolds numbers of about $1.15-1.2 \cdot \operatorname{Re}_{c r}$. Next, as the Reynolds number increases, the reduced mixing efficiency decreases due to the increase in the pressure drop. That range of Reynolds numbers 1.15-1.2 $\operatorname{Re}_{c r}$. should be considered as optimal from the micromixer efficiency point of view.

Analysis of the influence of the micromixers relative sizes was shown that it is more advantageous to use mixers with high value of the dimensionless width of the mixing channel $\left(w / w_{0}>1.5\right)$ and moderate value of the dimensionless height of the mixing channel $\left(1 \leq h / h_{0} \leq 1.5\right)$ for optimal operation. In such configurations, additional vortices are formed in the mixing channel, that leading to an increase in the mixing efficiency with a decrease in pressure drop due to the increase in the channel cross-section. Such an analysis of the efficiency of the T-shaped micromixers was performed for the first time. 

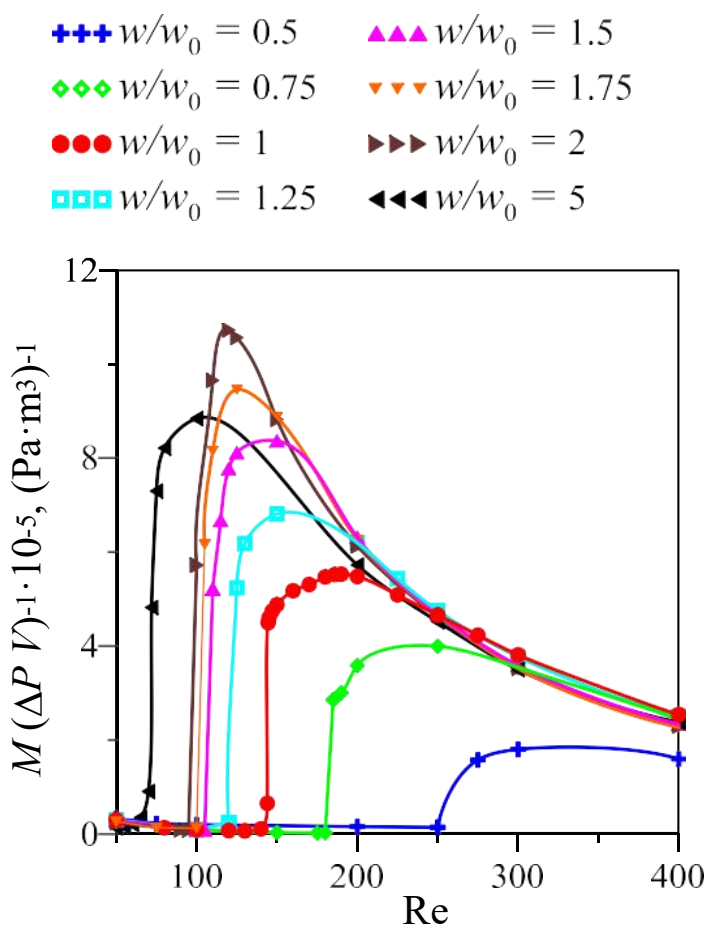

a)

$$
\begin{aligned}
& \boldsymbol{+ + +} h / h_{0}=0.5 \quad \Delta \boldsymbol{\Delta} \mathbf{\Delta} / h_{0}=1.5 \\
& \diamond \diamond \diamond h / h_{0}=0.75 \quad \vee \vee \vee h / h_{0}=2.5 \\
& \bullet h / h_{0}=1 \quad \longmapsto h / h_{0}=5 \\
& \text { 口an } h / h_{0}=1.25 \quad \text { ४४ } h / h_{0}=10
\end{aligned}
$$

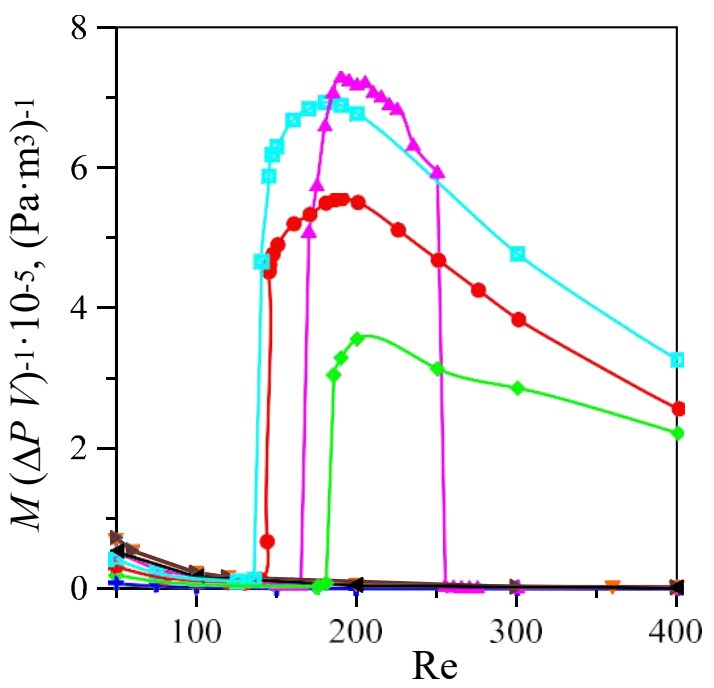

b)

Figure 17. The dependence of the mixing efficiency in the mixer related to pressure drop and to the volume of fluid in the mixing channel on the Reynolds number

\section{CONCLUSIONS}

The numerical investigation of fluids flow regimes and mixing modes in T-shaped microchannels with different geometrical dimensions of the mixing channel was carried out. The dimensionless width and height of the mixing channel were varied in wide ranges: $0,5<w / w_{0}<5$ и $0,5<h / h_{0}<10$. Much attention is given to the influence of the geometrical dimensions of the mixer on the critical value of the Reynolds number, corresponding to the transition from the steady symmetric to the asymmetric (engulfment) flow regime. That regime is most interesting for practice since the mixing efficiency increases tens of times in such regime.

As a result of calculations, it was found that the engulfment flow regime is observed in all considered range of the dimensionless width of the mixing channel. A strict dependence of the critical Reynolds number on the width of the mixing channel was found. It was shown that as the channel width increases that value decreases monotonously.

It was found, that as the dimensionless height of the mixing channel varies, the onset of the engulfment regime is observed only for configurations of the mixers with the mixing channel heights in the range from $0.75 h_{0}$ to $1.5 h_{0}$. It was obtained that the key factor in the transition from symmetric to asymmetric flow regime is the distance between the vortices 
formed at the upper and lower walls of the mixing channel. The engulfment flow regime is observed only in those channels, in which the distance between the vortices, on the one hand, is greater than necessary for the existence of two vortices and, on the other hand, is sufficient for their interaction. This explains the fact that the dependence of the critical Reynolds number on the dimensionless channel height has a minimum (see Fig. 13b).

Besides, in contrast to most previous studies, a systematic study of the pressure drop in the T-shaped microchannel in a wide range of its geometric dimensions was carried out. The dependences of the friction factor on the Reynolds number and the geometric dimensions of the mixer are established. Based on the carried out studies, the correlation dependencies that make it possible to reproduce the pressure drop in the micromixer with an error that not exceeding $5 \%$ in the entire range of considered parameters were proposed.

It was shown that the previously known correlation from [24] for determining the critical Reynolds number works only in a narrow range of the dimensionless width and height of the mixing channel: $0.5<w / w_{0}<2$ and $0.5<h / h_{0}<1$. To determine the critical Reynolds number in a wider range of parameters, a new correlation (5) that describes the calculated data with an error that not exceeding $5 \%$ was proposed.

The obtained data on mixing efficiency and pressure losses in the channel allowed to determine the optimal modes of the T-shaped micromixer for the first time. To analyze the mixing efficiency in micromixers of various sizes, it is suggested to use the mixing efficiency reduced to a unit of volume and $1 \mathrm{~Pa}$ of pressure loss. The analysis was shown that reduced mixing efficiency has a maximum in the region of the Reynolds numbers of about 1.151.2 $\mathrm{Re}_{c r}$. This range of Reynolds numbers $1.15-1.2 \cdot \mathrm{Re}_{c r}$. should be considered as optimal from the efficiency of the micromixer point of view.

The work is performed at partial financial support of the Russian Science Foundation grant (project No. 16-19-10519).

\section{REFERENCES}

1. Tabeling $P$. Introduction to microfluidics. Oxford: Oxford University Press, 2005

2. Karnidakis G., Beskok A., Aluru N. Microflows and nanoflows // Interdisciplinary Applied Math. Springer Science+Business Media Inc., 2005. V. 29. 817 p.

3. Karnik R. Microfluidic mixing // Encyclopedia of microfluidics and nanofluidics / Ed. Li D. Springer, 2008. P. 1177

4. Rudyak V., Minakov A. Modeling and optimization of Y-type micro-mixers // Micromachines, 2014. Vol. 5. Is. 4. Pp. 886-912 
5. Mansur E., Mingxing Y., Yundong W., Youyuan D. A state-of-the-art review of mixing in microfluidic mixers // Chinese J. Chem. Eng. 2008. Vol. 16. Is. 4. Pp. 503-516

6. Hoffmann M., Schluter M., Rubiger N. Experimental investigation of fluid-fluid mixing in T-shaped micro-mixers using $\mu$-LIF and $\mu$-PIV // Chem. Eng. Sci., 2006. Vol. 61. Is. 9. Pp. 2968-2976

7. Bothe D., Stemich C., Warnecke H.-J. Theoretische und Experimentelle Untersuchungen der Mischvorgänge in T-förmigen Mikroreaktoren - Teil 1: Numerische Simulation und Beurteilung des Strömungsmischens // CIT, 2006. Vol. 76. Is. 10. Pp. 1480-1484

8. Bothe D., Stemich C., Warnecke H.-J. Fluid mixing in a T-shaped micro-mixer // Chem. Eng. Sci., 2006. V. 61. Is. 9. Pp. 2950-2958

9. Stemich $C$. Theoretische und numerische Untersuchung des Struomungsmischens in einem T-formigen Mikromischer. Dissertation zur Erlangung des akademischen Grades Doktor der Naturwissenschaften. Universit at Paderborn, 2006.

10. Dreher S., Kockmann N., Woias P. Characterization of laminar transient flow regimes and mixing in T-shaped micro-mixers // Heat Trans. Eng., 2009. Vol. 30. Is. 1-2. Pp. 91-100

11. Minakov A.V., Rudyak V. Ya., Gavrilov A. A., Dekterev A.A. Mixing in T-shaped micromixer at moderate Reynolds numbers // Thermophysics and Aeromechanics, 2012. Vol. 19. Is. 3. Pp. 385-395

12. Minakov A., Rudyak V., Dekterev A., Gavrilov A. Investigation of slip boundary conditions in the T-shaped microchannel // Int. J. of Heat and Fluid Flow, 2013. Vol. 43. Pp. 161-169

13. Minakov A., Yagodnitsina A., Lobasov A., Rudyak V., Bilsky A. Study of fluid flow in micromixer with symmetrical and asymmetrical inlet conditions // La Houille Blanche, 2013. Is. 5. Pp. 12-21

14. Lobasov A.S., Minakov A.V., Rudyak V.Ya. Viscosity effect on the flow modes in T-type micro-mixers // Fluid Dynamics, 2016. Vol. 51. Is. 3. Pp. 381-388

15. Lobasov A.S., Minakov A.V. Density effect on the mixing efficiency and flow modes in Tshaped micromixers // MATEC Web of Conferences, 2017, Vol. 115. art. no. 07002

16. Lobasov A.S., Shebeleva A.A. Initial temperatures effect on the mixing efficiency and flow modes in T-shaped micromixer // IOP Conf. Series: Journal of Physics: Conf. Series, 2017, Vol. 899, art. no. 022010.

17. Galletti C., Arcolini G., Brunazzi E., Mauri R. Mixing of binary fluids with compositiondependent viscosity in a T-shaped micro-device // Chem. Eng. Sci., 2015. Vol. 123. Pp. 300-310 
18. Galletti C., Brunazzi E., Mauri R. Unsteady mixing of binary liquid mixtures with composition-dependent viscosity // Chem. Eng. Sci., 2017. Vol. 164. Pp. 333-343

19. Lobasov A.S., Minakov A.V. Analyzing mixing quality in a T-shaped micromixer for different fluids properties through numerical simulation // Chemical Engineering \& Processing: Process Intensification, 2018, Vol. 124, pp. 11-23

20. Orsi G., Roudgar M., Brunazzi E., Galletti C, Mauri R. Water-ethanol mixing in T-shaped microdevices // Chem. Eng. Sci., 2013. Vol. 95. Pp. 174-183

21. Wang W., Zhao S., Shao T., Jin Y., Cheng Y. Visualization of micro-scale mixing in miscible liquids using $\mu$-LIF technique and drug nano-particle preparation in T-shaped micro-channels // Chem. Eng. J.. 2012. Vol. 192. Pp. 252-261

22. Poole R.J., Alfateh M., Gauntlett A.P. Bifurcation in a T-channel junction: Effects of aspect ratio and shear-thinning // Chem. Eng. Sci., 2013. Vol. 104. Pp. 839-848

23. Andreussi T., Galletti C., Mauri R., Camarri S., Salvetti M.V. Flow regimes in T-shaped micro-mixers // Comp. and Chem. Eng., 2015. Vol. 76. Pp. 150-159

24. Soleymani A., Yousefi H., Turunen I. Dimensionless number for identification of flow patterns inside a T-micromixer// Chem. Eng. Sci., 2008. Vol. 63. Pp. 5291-5297

25. Fani A., Camarri S., Salvetti M.V. Investigation of the steady engulfment regime in a three-dimensional T-mixer // Phys. Fluids, 2013. Vol. 25. Is. 6. 064102

26. Fani A., Camarri S., Salvetti M.V. Unsteady asymmetric engulfment regime in a T-mixer // Physics of Fluids, 2014. Vol. 26. Is. 7. 074101

27. Galletti C., Roudgar M., Brunazzi E., Mauri R. Effect of inlet conditions on the engulfment mode in a T-shaped micro-mixer // Chem. Eng. J., 2012. Vol. 185. Pp. 300313.

28. Silva J.P., Santos A., Semiao V. Experimental characterization of pulsed Newtonian fluid flows inside T-shaped micromixers with variable inlets widths // Experimental Thermal and Fluid Science, 2017. Vol. 89. Pp. 249-258.

29. Cortes-Quiroz C.A., Azarbadegan A., Zangeneh M. Effect of channel aspect ratio of 3-D T-mixer on flow patterns and convective mixing for a wide range of Reynolds number // Sensors and Actuators B: Chemical, 2017. Vol. 239. Pp. 1153-1176

30. Basavarajappa M., Elsnab J., Thomas S., Ameel T.A. Experimental investigation of mixing in a T-channel for asymmetric inlet conditions // Proceedings of the ASME 2012 10th International Conference on Nanochannels, Microchannels and Minichannels (ICNMM2012), July 8-12, 2012, Rio Grande, Puerto Rico 
31. Hossain S., Kim K.-Y. Mixing Performance of a Serpentine Micromixer with Non-Aligned Inputs // Micromachines, 2015. Vol. 6. Pp. 842-854.

32. Cherlo S.K.R., Pushpavanam S. Effect of depth on onset of engulfment in rectangular micro-channels // Chem. Eng. Sci. 2010. 65, 6486-6490.

33. Gavrilov A.A., Minakov A.V., Dekterev A.A., Rudyak V.Ya. A numerical algorithm for modeling laminar flows in an annular channel with eccentricity // J. of App. and Ind. Math., 2011. Vol. 5. Is. 4. Pp. 559.-568

34. Patankar $S$. Numerical methods in heat transfer and fluid dynamics. M.: Energoatomizdat, 1984.

35. Bystrov Yu. A., Isaev S.A., Kudryavtsev N.A. Leont'ev A.I. Numerical simulation of vortex based enhancement of heat transfer in tube packages. Shipbuilding, 2005.

36. Ferziger J.H., Peric M. Computational methods for fluid dynamics. Berlin: Springer Science+Business Media Inc., 2002.

37. Leonard B.P. A stable and accurate convective modeling procedure based on quadratic upstream interpolation // Comp. Math. Appl. Mech. Eng., 1979. Vol. 19. Is. 1. Pp. 59-98

38. Rhie C.M., Chow W.L. Numerical study of the turbulent flow past airfoil with trailing edge separation // AIAA Journal, 1983. Vol. 21. Is. 11. Pp. 1525-1532

39. Trottenberg U., Oosterlee C.W., Schüller A. Multigrid. Academic Press, 2001.

40. Ligrani P.M. A Study of Dean Vortex Development and Structure in a Curved Rectangular Channel With Aspect Ratio of 40 at Dean Numbers up to 430 / Prepared for Vehicle Propulsion Directorate U.S. Army Research Laboratory (Contractor Report ARLCR-144) and Lewis Research Center under NASA Defense Purchase Requests (Contractor Report 4607) MIPR DOC NR C-80019-F and MIPR DOC NR C-30030-P, 1994, 226 P. 